\title{
Vascular parkinsonism and vascular dementia are associated with an increased risk of vascular events or death
}

\author{
Jacek Staszewski ${ }^{1}$, Renata Piusińska-Macoch ${ }^{1}$, Bogdan Brodacki ${ }^{1}$, Ewa Skrobowska ${ }^{2}$, \\ Katarzyna Macek ${ }^{1}$, Adam Stępień ${ }^{1}$
}

\begin{abstract}
${ }^{1}$ Clinic of Neurology, Military Institute of Medicine, Warsaw, Poland
${ }^{2}$ Department of Radiology, Military Institute of Medicine, Warsaw, Poland
\end{abstract}

Submitted: 1 June 2017

Accepted: 10 June 2017

Arch Med Sci Atheroscler Dis 2017; 2: e16-e23

DOI: https://doi.org/10.5114/amsad.2017.68549

Copyright (c) 2017 Termedia \& Banach

\section{Abstract}

Introduction: The natural course of vascular parkinsonism (VaP) and dementia (VaD) due to cerebral small vessel disease (SVD) is not well known. The aim of this single-center study was to evaluate the long-term risk of vascular events, death and dependency in patients with VaP or VaD and to compare it with patients without cerebrovascular disease but with high atherothrombotic risk.

Material and methods: Seventy-eight consecutive, functionally independent patients with MRI features of SVD and with recently diagnosed VaD $(n=50)$ and $\operatorname{VaP}(n=28)$ and 55 controls (control group - CG) with high 10-year risk of total cardiovascular disease (SCORE $\geq 5 \%$ ) were prospectively recruited and followed for 24 months.

Results: Patients with SVD had lower prevalence of coronary artery disease compared with the CG $(20.5 \%$ vs. $40 \% ; p=0.02)$ but similar prevalence of other atherothrombotic risk factors including mean age $(73.7 \pm 7.3$ vs. 72 \pm 5.9 years, $p=0.09$ ). All outcomes were worse in SVD patients than controls. Thirty-one percent of SVD patients ( $34 \%$ of $\mathrm{VaD}$ Vs. $25 \%$ of $\mathrm{VaP}, p=0.45$ ) experienced vascular events or died compared to $6 \%$ of controls $(p<0.01)$. After adjustments for potential confounders (age, sex, vascular risk factors), patients with $\mathrm{VaP}(\mathrm{HR}=7.5 ; 95 \% \mathrm{Cl}: 1.6-33 ; p<0.01)$ and $\mathrm{VaD}(\mathrm{HR}=8.7$; $95 \% \mathrm{Cl}: 2.1-35 ; p<0.01)$ had higher risk of vascular events or death and death or dependency (respectively; $\mathrm{HR}=3.9 ; 95 \% \mathrm{Cl}: 0.83-18.8 ; p=0.07$ and $\mathrm{HR}=4.7,95 \% \mathrm{Cl}: 1.1-19.7 ; p=0.03$ ).

Conclusions: Patients with VaP or VaD due to SVD had significantly higher risk of vascular events, death and dependency compared to controls with high cardiovascular risk and without cerebrovascular disease.

Key words: cerebral small vessel disease, mortality, vascular events, prognosis, vascular parkinsonism, vascular dementia

\section{Introduction}

Cerebral small vessel disease (SVD) is one of the most important and common vascular diseases of the brain, caused by lacunar infarcts, white matter lesions (WMLs), microbleeds and intraparenchymal hemorrhage in the cerebral white and deep gray matter [1]. Small vessel disease can cause several different types of distinct or overlapping clinical presentations, including vascular dementia $(\mathrm{VaD})$, parkinsonism $(\mathrm{VaP})$ or re-

\author{
Corresponding author: \\ Jacek Staszewski \\ Clinic of Neurology \\ Military Institute of Medicine \\ 128 Szaserow St \\ 04-141 Warsaw, Poland \\ Phone: +48 261816445 \\ Fax: +48 228106100 \\ E-mail: jstaszewski@wim.mil.pl
}


current strokes. The prognosis of SVD, especially in chronic symptomatic SVD patients, e.g. VaP or $\mathrm{VaD}$, is not well known, and the risk of vascular events remains controversial [2].

In this single-center case-control study, we prospectively evaluated the long-term risk of vascular events or death in patients with non-disabling VaP and $\mathrm{VaD}$ compared with patients without cerebrovascular disease but with high atherothrombotic risk.

\section{Material and methods}

The present investigation is embedded in the SHEF-SVD Study (Significance of HEmodynamic and hemostatic Factors in the course of different manifestations of Cerebral Small Vessel Disease) [3]. The study group consisted of 78 patients: 28 with VaP and 50 with subcortical VaD considered to be caused by SVD, and 55 controls (CG). We included patients with $\mathrm{VaP}$ or $\mathrm{VaD}$ as these symptoms usually overlap in advanced stages of SVD and to the best of our knowledge no study has compared their clinical course based on uniform methodology. The patients were recruited from the General or Neurological Outpatient Department and prospectively enrolled in the study between December 2011 and June 2014. The study protocol and methods have been thoroughly described elsewhere [3].

In brief, the SVD group consisted of consecutive patients with a newly diagnosed presumed $\mathrm{VaD}$ or VaP, with evidence of SVD findings on neuroimaging, who were independent and had no severe dementia (Mini-Mental State Examination (MMSE) $\geq 12$ points). The patients were diagnosed according to the typical radiological and clinical picture, after exclusion of other neurodegenerative conditions using clinical tools easily applied in clinical practice: Hurtig criteria or NINDS-AIREN criteria with Modified Hachinski Ischemic Scale $\geq 7$ points, respectively [4-6]. Patients with previous stroke, strategic single-infarct dementia or with post-stroke $\mathrm{VaD}$ or $\mathrm{VaP}$ were excluded. The date of the first symptoms related to the $\mathrm{VaP}$ and $\mathrm{VaD}$ was used to establish the exact start date of these diseases. The control group consisted of patients with normal brain magnetic resonance imaging (MRI), without history of cerebrovascular disease and with high cardiovascular risk assessed according to the European Society of Cardiology and the European Atherosclerosis Society Guidelines (2011) [7]. High risk was recognized in patients with: documented cardiovascular disease (CVD) previous myocardial infarction (MI), acute coronary syndrome, coronary revascularization/bypass graft or peripheral arterial disease (PAD); diabetes (type 2 or type 1 diabetes with target organ damage e.g. microalbuminuria); moderate to severe chronic kidney disease (CKD; glomerular filtration rate (GFR)
< $60 \mathrm{ml} / \mathrm{min} / 1.73 \mathrm{~m}^{2}$ ); or markedly elevated single risk factors such as familial dyslipidemias and severe hypertension (systolic blood pressure (SBP) $\geq 180 \mathrm{~mm} \mathrm{Hg}$ and/or diastolic blood pressure (DBP) $\geq 110 \mathrm{~mm} \mathrm{Hg}$ ); or 10-year risk of total CVD $\geq 5 \%$ (estimated using the Systemic Coronary Risk Estimation (SCORE) risk assessment charts according to gender, smoking status, age, blood pressure and total cholesterol (TC)) [8]. Participants from both groups were aged between 60 and 90 years. Patients with significant stenosis ( $>50 \%$ ) of a major extracranial or intracranial artery, atrial fibrillation, non-SVD related WMLs (e.g. due to migraine, vasculitis, multiple sclerosis, CADASIL), life expectancy of less than 6 months, and MRI contraindications were excluded.

\section{Procedure}

Detailed neurological examination and assessments of cognitive function (MMSE), disability (Barthel index, $\mathrm{BI}$ ) and parkinsonism disease stage (Hoehn-Yahr) were performed at baseline and after 24 months. Patients were regarded as dependent if they gained $\leq 80$ points in total $\mathrm{BI}$ scores [9]. Based on medical records, physical examination and comprehensive history available at baseline, we evaluated atherothrombotic risk factors, including tobacco use, diabetes, hyperlipidemia, hypertension, coronary artery disease (CAD), peripheral vascular disease and CKD. All patients received optimal medical treatment, according to guidelines. Data regarding vascular events during the study flow and/or cause of death (classified according to the ICD-10) were obtained from the treating physician, and/or general practitioner or medical records. Patients with incomplete follow-up were censored at the last observation.

For MRI evaluation we used imaging with standard T2-weighted, FLAIR and gradient echo sequences. Periventricular and deep WMLs were visualized on $T 2$ and PD/FLAIR images as ill-defined hyperintensities $\geq 5 \mathrm{~mm}$. The simple modified Fazekas rating scale was used to estimate the extent of the periventricular and deep WMLs [10]. Mild WMLs (grade 1) was defined as punctate lesions in the deep white matter with a maximum diameter of $9 \mathrm{~mm}$ for a single lesion and of $20 \mathrm{~mm}$ for grouped lesions. Moderate WMLs (grade 2) were early confluent lesions of 10-20 mm single lesions and $>20 \mathrm{~mm}$ grouped lesions in any diameter, and no more than connecting bridges between the individual lesions. Severe WMLs (grade 3) were single lesions or confluent areas of hyperintensity of $\geq 20 \mathrm{~mm}$ in any diameter. All patients in the SVD group had at least grade 1 WMLs; those in the CG were included only following normal MRI. All MRI scans were obtained using the same scanner (GE Healthcare 1.5T scanner) and evalu- 
ated by the same experienced investigator (E.S.) blinded to the clinical diagnosis.

The main outcome event was time to any vascular event (stroke - hemorrhagic or ischemic, myocardial infarction or peripheral vascular intervention) or death. Vascular death included death resulting from an acute $\mathrm{MI}$, sudden cardiac death, heart failure, stroke or cardiovascular procedures or hemorrhage, and death due to other CVD [11] Non-cardiovascular death was defined as any death with a specific cause that is not thought to be CVD in nature. Undetermined cause of death referred to a death not attributable to one of the above categories. Ischemic (IS) and hemorrhagic strokes were defined based on typical radiological and clinical features with focal cerebral, spinal, or retinal dysfunction. Myocardial infarction was defined as a clinical or pathologic event caused by myocardial ischemia in which there was evidence of myocardial injury or necrosis with a rise and/ or fall of cardiac biomarkers, along with supportive evidence in the form of typical symptoms, suggestive electrocardiographic changes, or imaging evidence of new loss of viable myocardium or new regional wall motion abnormality. Peripheral vascular intervention was defined as a catheter-based or open surgical procedure designed to improve arterial or venous blood flow or otherwise modify or revise vascular conduits.

This study complied with the Declaration of Helsinki. All participants from both groups signed an informed consent form. This study was approved by the local Medical Ethics Committee.

\section{Statistical analysis}

Log-normal data were compared using paired t-tests, non-normal data were analyzed using nonparametric tests, and the $\chi^{2}$ test was used for comparisons of categorical variables. The difference between baseline risk factors was compared between SVD and CG and between $\mathrm{VaD}$ and VaP groups. The Kaplan-Meier method was used to estimate the event-free survival, and study groups were compared using Cox regression. Adjusted hazard ratios (HRs) and $95 \% \mathrm{Cls}$ were calculated with fixed entry of a pre-defined set of potential confounders (age, sex, vascular risk factors) selected on the basis of clinical plausibility, previous literature reviews and measured at the baseline visit. Dead or dependent at 24-month follow-up was analyzed using Cox logistic regression. We did not control for baseline WMLs because it may distort estimates of change in longitudinal studies. A probability value of $p<0.05$ was considered significant. All data are presented as mean \pm SD values. All analyses were performed using Statistica 12 software (StatSoft Inc, USA).

\section{Results}

\section{Patient characteristics}

The study included 133 patients (78 with SVD and 55 in the CG). All controls had high CVD risk: 35 (63\%) patients had documented symptomatic large artery disease (CAD or PAD), $3(5.5 \%)$ had diabetes and CKD, 2 (3.6\%) had diabetes alone, and the remaining 15 (27\%) patients had elevated 10-year risk of CVD (SCORE $\geq 5 \%$ ) caused by other cardiovascular risk factors (Table I). No patients from either group had severe hypertension or familial dyslipidemia. Patients with SVD were less likely to have CAD and had non-significantly higher prevalence of diabetes and polymetabolic syndrome compared to controls. Mean time from first symptoms to enrollment was $25.5 \pm 11$ months in $\mathrm{VaD}$ and $27.5 \pm 14$ months in $\operatorname{VaP}(p=0.7)$. The majority of patients with SVD (80.8\%) had moderate or severe WMLs on baseline MRI $(78 \%$ in $\mathrm{VaD}$ and $82.2 \%$ in VaP; $p=0.6)$. The mean Hoehn-Yahr stage at baseline in patients with VaP was 2.6 \pm 0.85 , and 23 (82\%) patients were classified in Hoehn-Yahr stage 3.

\section{All-cause mortality and vascular events}

Information on the outcome was available from $97 \%$ of the enrolled patients; 4 patients ( 2 in $\mathrm{VaD}$ and 2 in $\mathrm{CG}$ ) were lost to follow-up. The mean follow-up time in the SVD group was $23.1 \pm 4$ months (150.15 person-years): $23.7 \pm 3.4$ in $\mathrm{VaD}$ (98.8 person-years), $22 \pm 4.6$ months (51.3 person-years); $p=0.7$ and $23.4 \pm 3$ months (109.2 person-years) in the CG $(p=0.2)$. There were 17 nonfatal vascular events in the SVD group (16 ischemic strokes, $1 \mathrm{MI}$ ) and 3 in the CG (1 IS, $2 \mathrm{MIs})$. Seven patients with SVD (9\%) and none in the CG died during follow-up $(p=0.01)$. The cause of death was vascular related in 4 (57\%) patients (cardiac cause, $n=4$ ), nonvascular in $3(43 \%)$ (infection, $n=1$; malignant neoplasm, $n=1)$ and undetermined in 1 patient. The majority $(91 \%)$ of patients with SVD who experienced the main outcome had grade 2 or 3 WMLs: $93 \%$ in $\mathrm{VaD}$ and $85.7 \%$ in VaP. Kaplan-Meier survival curves for the SVD group showed significantly higher risk compared with the CG (log rank $p=$ 0.01) (Figure 1). In a Cox regression model after adjustments for potential confounders (age, sex, hypertension, diabetes, hypercholesterolemia, smoking, $(A D)$ the only independent predictors for the main outcome were the presence of $\mathrm{VaD}$ $(\mathrm{HR}=8.7 ; p<0.01), \mathrm{VaP}(\mathrm{HR}=7.5 ; p=0.01)$ and hypertension $(\mathrm{HR}=1.4 ; p=0.04)$, while there was no significant difference in risk of study outcome between $\mathrm{VaD}$ and $\mathrm{VaP}(\mathrm{HR}=1.17 ; 95 \% \mathrm{Cl}$ : $0.39-3.5 ; p=0.7$ ) (Table II). 
Table I. Baseline characteristics of patients with cerebral small vessel disease (SVD) and controls (CG)

\begin{tabular}{|c|c|c|c|c|c|c|}
\hline Parameter & SVD & CG & $P$-value & VaD & VaP & $P$-value \\
\hline$N(\%)$ & 78 & $55(27)$ & - & $50(33)$ & $28(19)$ & - \\
\hline Age, mean $( \pm S D)$ years & $73.7(7.3)$ & $72.0(5.9)$ & 0.09 & $74.4(7.9)$ & $72.3(6.2)$ & 0.2 \\
\hline Female sex, $n(\%)$ & $47(60.3)$ & $25(45.5)$ & 0.11 & $37(74)$ & $10(35.7)$ & $<0.01$ \\
\hline Hypertension & $12(21.8)$ & $43(78.2)$ & 0.65 & $44(88)$ & $20(71.4)$ & 0.12 \\
\hline Coronary artery disease & $16(20.5)$ & $22(40)$ & 0.02 & $13(26)$ & $3(10.7)$ & 0.14 \\
\hline Diabetes mellitus & $42(53.8)$ & $20(37)$ & 0.08 & $28(56)$ & $14(50)$ & 0.64 \\
\hline Current smoking & $22(28.2)$ & $15(27.3)$ & 0.9 & $11(22)$ & $11(39.3)$ & 0.12 \\
\hline Hyperlipidemia & $57(73.1)$ & $43(78)$ & 0.54 & $37(74)$ & $20(71.4)$ & 0.79 \\
\hline $\begin{array}{l}\text { Peripheral arterial } \\
\text { disease }\end{array}$ & $10(12.8)$ & $13(23.6)$ & 0.16 & $4(8)$ & $6(21.4)$ & 0.15 \\
\hline $\mathrm{BMI}\left[\mathrm{kg} / \mathrm{m}^{2}\right]$ & $26.4(4.3)$ & $26.4(4)$ & 0.9 & $27(4.5)$ & $25.4(3.6)$ & 0.12 \\
\hline $\begin{array}{l}\text { Polymetabolic } \\
\text { syndrome }\end{array}$ & $30(38.5)$ & $13(23.6)$ & 0.09 & $21(42)$ & $9(32.1)$ & 0.47 \\
\hline Obesity (BMI > 30 kg/m²) & $17(21.8)$ & $11(20)$ & 0.83 & $12(24)$ & $5(17.9)$ & 0.58 \\
\hline CKD & $10(12.8)$ & $3(5.5)$ & 0.23 & $5(10)$ & $5(17.9)$ & 0.48 \\
\hline Total cholesterol [mg/dl] & $188.1(44.2)$ & $192.2(38.9)$ & 0.59 & $188.5(45.3)$ & $187.5(43.1)$ & 0.92 \\
\hline $\mathrm{SBP} / \mathrm{DBP}[\mathrm{mm} \mathrm{Hg}]$ & $\begin{array}{c}131.5(14.9) / \\
75.4(10)\end{array}$ & $\begin{array}{c}125.3(18) / \\
74.8(8.2)\end{array}$ & $0.14 / 0.8$ & $\begin{array}{c}132.9(15.8) / \\
77.1(9.7)\end{array}$ & $\begin{array}{c}128.8(13.1) / \\
72.2(10.3)\end{array}$ & $0.43 / 0.15$ \\
\hline TG [mg/dl] & $117.2(49.7)$ & $125.8(142)$ & 0.63 & $111.8(46.6)$ & $126.3(54.1)$ & 0.23 \\
\hline $\mathrm{BI}$ & $93.8(13.5)$ & $93.5(16.12)$ & 0.9 & $92.4(14.4)$ & $96.2(11.9)$ & 0.2 \\
\hline MMSE & $22(7.2)$ & $25(2)$ & $<0.01$ & $21.5(8.6)$ & $22.9(3.3)$ & 0.4 \\
\hline Antiplatelet treatment & $56(71.8)$ & $24(43.6)$ & 0.001 & $37(74)$ & $19(67.9)$ & 0.6 \\
\hline Statin treatment & $53(67.9)$ & $31(57.4)$ & 0.27 & $36(72)$ & $17(60.7)$ & 0.32 \\
\hline \multicolumn{7}{|l|}{ Outcome: } \\
\hline $\begin{array}{l}\text { Vascular events or } \\
\text { death }\end{array}$ & $24(30.8)$ & $3(5.5)$ & $<0.01$ & $17(34)$ & $7(25)$ & 0.45 \\
\hline Total deaths & $7(9)$ & 0 & $0.01^{\#}$ & $3(6)$ & $4(14.3)$ & $0.35^{\#}$ \\
\hline IS & $16(20.5)$ & $1(1.8)$ & $<0.01$ & $14(28)$ & $2(7.1)$ & 0.04 \\
\hline MI & $1(1.3)$ & $2(3.6)$ & 0.56 & 0 & $1(3.6)$ & 0.35 \\
\hline $\begin{array}{l}\text { Dead or dependent } \\
\text { at follow-up* }\end{array}$ & $21(27.6)$ & $5(9.4)$ & 0.04 & $16(35)$ & $5(20)$ & 0.1 \\
\hline $\begin{array}{l}\text { Institutionalized } \\
\text { during follow-up** }\end{array}$ & $14(20)$ & $4(7.5)$ & 0.21 & $11(24)$ & $3(12.5)$ & 0.2 \\
\hline $\mathrm{BI}$ & $89.7(18.8)$ & 93.8 (12) & 0.19 & $88.2(20.2)$ & $92.6(88.7)$ & 0.39 \\
\hline MMSE & $24.3(3.1)$ & $19.2(6.1)$ & $<0.001$ & $18.3(6.5)$ & 22.7 (3.7) & $<0.001$ \\
\hline
\end{tabular}

Values are means ( $\pm S D$ ) or numbers of patients (\%). SVD - small vessel disease, CG - control group, VaD - vascular dementia, VaP vascular parkinsonism, BMI - body mass index, BI - Barthel index, CKD - chronic kidney disease, IS - ischemic stroke, MI - myocardial infarction, TG - triglycerides, MMSE - Mini Mental State Examination; *missing $n=2 \mathrm{VaD}, n=2 \mathrm{CG}$; ${ }^{* *}$ excluded 4 missing and 7 deceased patients; ${ }^{K}$ Kaplan-Meier log rank.

\section{Dependence and institutionalization at 2 years}

Almost one fifth ( $n=14 ; 19.7 \%)$ of SVD patients who survived required help from others in basic activities of daily living at 2 years compared to about $10 \%(n=5)$ of controls. Patients with VaD were non-significantly more frequently dependent compared with VaP at follow-up visit (respectively, $35 \%$ vs. $20 \%, p=0.1$ ) but the number of institutionalized patients was similar across groups. 


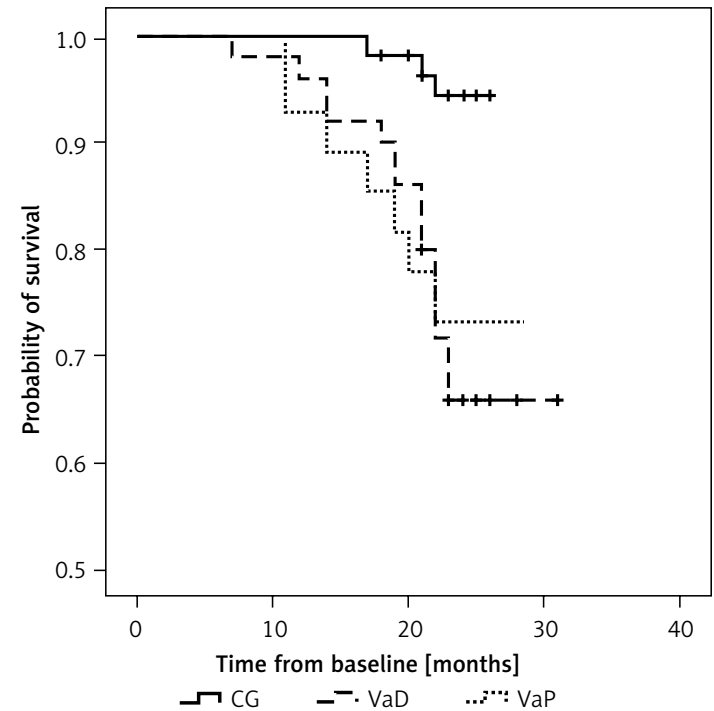

Figure 1. Kaplan-Meier curves for all-cause mortality and non-fatal vascular events by grouped diagnostic category (log rank, $p<0.01)$

$C G$ - control group, VaD - vascular dementia, VaP - vascular parkinsonism

Presence of $\mathrm{VaD}(\mathrm{HR}=4.7 ; p=0.03)$ and $\mathrm{VaP}$ $(\mathrm{HR}=3.9 ; p=0.07)$ were the only independent predictors for death and dependency after adjustments for confounders (Table II). Of those who survived, significantly $(p=0.03)$ more patients with $\mathrm{VaD}$ deteriorated in MMSE during follow-up ( $n=24 ; 53 \%$ ) compared to 6 patients with VaP (25\%), but almost half of patients with $\operatorname{VaP}(n=11$;
48\%) transited between Hoehn-Yahr stages during the study (mean: $3.4 \pm 0.7$ ).

\section{Discussion}

The present case-control study documented that patients with VaP and VaD due to SVD had a similar unfavorable outcome with high longterm risk of vascular events or death when compared to controls without cerebrovascular disease but with otherwise high CVD risk. Risk for dependency or institutionalization was also high but similar for VaD or VaP patients.

Little is known about the natural course of VaP and VaD caused by SVD, which makes our data the more important. VaP accounts for the minority (3-12\%) of all cases of parkinsonism; however, because of problems with definition and the clinical and pathological heterogeneity, its epidemiology is difficult to determine and previous studies of $\mathrm{VaP}$ were conducted on small groups of patients [12]. Our study showed that patients with VaP had eight-fold higher risk of vascular events or death compared to controls. This is in line with one prospective follow-up study of an unselected incident cohort of 38 patients with $\mathrm{VaP}$ and 262 age-sex matched controls which revealed that patients with VaP had increased mortality $(\mathrm{HR}=$ 6.85 ) and a $96 \%$ rate of death or dependency at 3 years [13]. Another study showed that median survival was shorter and standardized mortality ratios were higher in VaP compared with Parkin-

Table II. Adjusted risk ratios for different outcomes by diagnostic group

\begin{tabular}{|lcccc|}
\hline Parameter & $\begin{array}{c}\text { Non-fatal vascular events } \\
\text { or death HR }(95 \% \mathrm{Cl})\end{array}$ & $\begin{array}{c}P \text {-value } \\
\text { SVD vs. CG }\end{array}$ & $\begin{array}{c}\text { Dead or dependent } \\
\text { at 2 years HR (95\% Cl) }\end{array}$ & $P$-value \\
\hline VaD vs. CG & $2.88(1.4-5.6)$ & 0.002 & $2.1(1.08-4)$ & 0.02 \\
\hline VaP vs. CG & $7.5(1.6-33)$ & 0.002 & $4.7(1.1-19.7)$ & 0.03 \\
\hline Age & $1.03(0.9-1.09)$ & 0.008 & $3.9(0.83-18.8)$ & 0.07 \\
\hline Sex: female & $0.77(0.3-1.8)$ & 0.57 & $1.01(0.93-1.08)$ & 0.87 \\
\hline Hypertension & $1.4(1.2-2.3)$ & 0.04 & $1.01(0.36-2.7)$ & 0.61 \\
\hline Diabetes & $0.88(0.4-1.9)$ & 0.75 & $1.1(0.29-4.1)$ & 0.88 \\
\hline Hypercholesterolemia & $1.2(0.8-1.9)$ & 0.25 & $1.2(0.32-4.6)$ & 0.41 \\
\hline Smoking & $0.64(0.2-1.7)$ & 0.38 & $1.3(0.46-4.36)$ & 0.76 \\
\hline CAD & $1.7(0.6-4.4)$ & 0.27 & $1.4(0.4-5.2)$ & 0.53 \\
\hline CKD & $0.5(0.1-1.7)$ & 0.5 & $0.8(0.2-2.1)$ & 0.54 \\
\hline Obesity & $1.4(0.32-5.7)$ & 0.41 & $1.68(0.51-5.4)$ & 0.43 \\
\hline Antiplatelet treatment & $0.73(0.27-1.9)$ & 0.52 & $0.46(0.19-2.4)$ & 0.38 \\
\hline Statin treatment & $0.82(0.27-2.47)$ & 0.72 & $0.69(0.19-2.4)$ & 0.56 \\
\hline
\end{tabular}

SVD - small vessel disease, CG - control group, VaD - vascular dementia, VaP - vascular parkinsonism, CAD - carotid artery disease, CKD - chronic kidney disease. 
son's disease (2.1 vs. 7.8 years and 4.2 vs. 1.5, respectively) [14].

The risk of all-cause death in patients with $\mathrm{VaD}$ (6\%) was lower compared to a study in which $24 \%$ of individuals with $\mathrm{VaD}$ followed for 2 years died [15]. An even more pronounced risk $(R R=11)$ was reported previously when patients with different types of dementia were compared with controls, which may be, however attributed to their significantly older age than in the current study (mean: 85 vs. 74 years) [16]. The important causes of death in these patients compared to the general population were dementia alone and cerebrovascular diseases. In another study, only $39 \%$ of patients with $\mathrm{VaD}$ survived 5 years as compared with $75 \%$ of age-matched controls, presumably because of the coexistence of other vascular risk factors [17]. Similar to our results, one study showed that cardiac diseases were responsible for $55 \%$ of causes of death in patients with $\mathrm{VaD}$ and respiratory system diseases (e.g., pneumonia) in $33 \%$, whilst the corresponding figures were $23 \%$ and $56 \%$ for patients with Alzheimer type of dementia [18]. Patients with different types of dementia have also an increased risk of hospitalization, including hospitalization for ambulatory care-sensitive conditions [19]. Exclusion of patients with atrial fibrillation and significant stenosis of extracranial or intracranial arteries in all patients in the presented study resulted in a low incidence of cardioembolic- or large vessel disease related vascular events, but the excess risk of nonvascular death in the SVD group can be explained. As these diseases progress, patients become increasingly frail, which gives rise to complications such as swallowing impairment, incontinence or falls; however, we did not find any injury-related death in our group of patients. Variability in clinical presentations and course of SVD may be attributable to either the control or the aftermath of traditional vascular risk factors, but in our study we did not observe much difference in prevalence of CVD risk factors between study groups.

The major difference in risk of vascular events between SVD and CG (respectively, 20.5\% vs. 9\%) was related to lacunar strokes (LS). All recurrent IS in the SVD group in our study were of lacunar type, supporting a distinctive pathomechanism. It suggests that the pathophysiology and course of SVD may be independent from those of atherosclerotic large artery disease and a "nonvascular" component with contributing mechanisms may play a role in the cause and long-term course of SVD [20]. These results are similar to studies evaluating prognosis of patients following $L S$ in which an excess of death and stroke recurrence has been consistently documented [21]. The risk of death immediately after stroke onset was low but increased with time, reaching $15 \%$ at 10 years, and $60 \%$ of the patients died after 10 years [22]. The rate of LS in VaP (7.1\%) was similar to rates of $5-11 \%$ reported in prior prospective and epidemiological studies [23, 24]. These results were also in line with the SPS3 trial, in which, depending on the blood pressure control, patients with recent LS experienced $8 \%$ to $10 \%$ of recurrent strokes and $6.6 \%-7 \%$ died during 3.7 years of follow-up [25]. It gave an annualized rate of recurrent stroke between $2.25 \%$ and $2.77 \%$ and death from all causes between $1.74 \%$ and $1.8 \%$. The rate of strokes in $\mathrm{VaD}$ (28\%) was however higher than in VaP, which is difficult to explain as the prevalence of risk factors and WMLs load were not different between studied groups, but it may result from either the low number of IS or recruited patients with VaP.

The unfavorable course of SVD can depend on several underlying mechanisms. Cerebral SVD reflects potential vulnerability of the brain and an augmented susceptibility to vascular brain damage. These changes may increase the risk of not only acute infarction but also chronic ischemia from reductions in perfusion and gas transfer in the white matter and deep brain structures $[1,26]$. It has been suggested that areas with extensive WMLs may be poorly supplied by collateral compensation, which may lead to IS extension, decreased neuronal connectivity and poor brain compensation and recovery [27]. It is interesting that even in the present study an apparent similarity emerged between the course of $\mathrm{VaD}$ and VaP, which can further prove common pathogenetic mechanisms of these entities. The majority of our patients with SVD had moderate or severe WMLs at baseline. The presence of severe WMLs is linked with a worse prognosis with high risk of vascular events and death. A meta-analysis and systematic review of 46 prospective studies revealed the doubled risk of death among patients with WMLs in comparison to those without [28]. There are also other neuroimaging markers of cerebral SVD, including cerebral microbleeds, which represent poor prognostic markers of recurrent vascular events and functional recovery for stroke survivors [29, 30]. Based on a series of cohort studies, the extent of WMLs increased the risk of stroke, myocardial infarction, and mortality in the community-dwelling general population [31]. The reason is not clear, as a precise distinction between the direct effects of SVD markers and bystander effects from shared vascular risk factors causing systemic micro- and macroangiopathies cannot be made. Major risk factors of VaP and $\mathrm{VaD}$ are principally the same as those of CVD, and their prevention and treatment appear to be of utmost importance. 
The results from our study suggest that regardless of the clinical manifestation, the course of chronic SVD is generally poor, as $28 \%$ of SVD patients (35\% VaD and $20 \% \mathrm{VaP})$ were dependent at the end of the study or died. These results are similar to patients following lacunar stroke in which $36 \%$ were reported to be dependent at 2 years and $42 \%$ at 3 years [26]. Also the LADIS study showed that the rate of transition from functional autonomy to disability after 1 year was $9 \%, 15 \%$ and $26 \%$ respectively in patients with grade 1 - WMLs [32].

Our study strengths include its prospective design and parallel 24-month observation of patients with well-characterized different SVD manifestations including rarely studied chronic VaP compared with the control population with high CVD risk sharing similar risk factors of microangiopathy. Another advantage is the complete follow-up for mortality, and single-center design, which allowed us to consistently collect all measures. Our study also has some limitations. The major weakness is the small number of patients and controls included. The total number of outcome events, especially in the CG, was relatively small. Although patients with $\mathrm{VaP}$ and $\mathrm{VaD}$ were included in the present study immediately after diagnosis, they were in an advanced stage of their disease; therefore it remains unknown whether our results can be applied to less severely affected patient. The overall study period (24 months) could ideally have been longer to generate more significant results. Therefore, future studies are needed to develop a predictive risk score for chronic manifestations of SVD, and to clarify the interaction between degenerative and vascular processes in their development. It would also be advisable for clinicians to identify highrisk patients to implement more effective preventive strategies. Precision in prediction of mortality could be improved e.g. by imaging modalities. As it is impossible to achieve $100 \%$ accuracy in intravital diagnosis of parkinsonism without pathological confirmation, there may be in our study, as well as in previous ones, some imprecision in that matter despite the fact that we applied strict diagnostic criteria based on all available clinical data.

In conclusion, the main outcome of our study is that VaP and VaD due to SVD are associated with an excess risk of death, increased rate of lacunar stroke and disability in comparison with controls with high CVD risk free of cerebrovascular disease. We also demonstrated that $\mathrm{VaD}$ and $\mathrm{VaP}$ shared similar unfavorable prognosis in the 24 months of observation. The precise mechanisms by which SVD mediates poor outcomes are unclear and require additional investigations. Further studies are warranted to evaluate the risk of SVD in the longer run.

\section{Acknowledgments}

This study was supported by the Polish Ministry of Science and Higher Education as a research project (N N402 473840).

\section{Conflict of interest}

The authors declare no conflict of interest.

\section{References}

1. Pantoni L. Cerebral small vessel disease: from pathogenesis and clinical characteristics to therapeutic challenges. Lancet Neurol 2010; 9: 689-701.

2. Norden AGW, Laat KF, Gons RAR. Causes and consequences of cerebral small vessel disease. The RUN DMC study: a prospective cohort study. Study rationale and protocol. BMC Neurol 2011; 11: 29.

3. Staszewski J, Piusińska-Macoch R, Skrobowska E, et al. Significance of haemodynamic and haemostatic factors in the course of different manifestations of cerebral small vessel disease: the SHEF-SVD Study - Study Rationale and Protocol. Neurosci J 2013; 2013: 424695.

4. Hurtig HI. Vascular parkinsonism. In: Parkinsonian syndromes. Stern MB, Koller WC (eds). Marcel Dekker, New York 1993; 81-3.

5. Chui HC, Victoroff JI, Margolin D. Criteria for the diagnosis of ischemic vascular dementia proposed by the State of California Alzheimer's Disease Diagnostic and Treatment Centers. Neurology 1992; 42: 473-80.

6. Zijlmans JCM, Daniel SE, Hughes AJ. Clinicopathological investigation of vascular parkinsonism, including clinical criteria for diagnosis. Mov Disord 2004; 19: 630-40.

7. ESC/EAS Guidelines for the management of dyslipidaemias. The Task Force for the management of dyslipidaemias of the European Society of Cardiology (ESC) and the European Atherosclerosis Society (EAS). Eur Heart J 2011; 32: 1769-818.

8. Kessler CS, Joudeth Y. Evaluation and treatment of severe asymptomatic hypertension. Am Fam Physician 2010; 15: 470-6.

9. Schulc E, Pallauf M, Mueller G, Wildbahner T, Themet C. Is the Barthel Index an adequate assessment tool for identifying a risk group in elderly people living at home? Int J Nurs Clin Pract 2015; 2: 140.

10. Fazekas F, Chawluk JB, Alavi A. MR signal abnormalities at $1.5 \mathrm{~T}$ in Alzheimer's dementia and normal aging. AJR Am J Roentgenol 1987; 149: 351-6.

11. Hicks KA, Hung HMJ, Mahaffey KW. Standardized Definitions for Cardiovascular and Stroke Endpoint Events in Clinical Trials. Draft Definitions for CDISC. 2014. Available at https://www.cdisc.org/therapeutic. Accessed August 20, 2014.

12. Morgante L, Salemi G, Meneghini F. Parkinson disease survival: a population-based study. Arch Neurol 2000; 57: 507-12.

13. Fielding S, Macleod AD, Counsell CE. Medium-term prognosis of an incident cohort of parkinsonian patients compared to controls. Parkinsonism Relat Disord 2016; 32: 36-41.

14. Macleod AD, Counsell CE. Mortality in Parkinson's disease and atypical Parkinsonian disorders [abstract]. Movement Disorders 2016; 30 Suppl 1.

15. van de Vorst IE, Koek HL, Bots ML. Evaluation of underlying causes of death in patients with dementia to sup- 
port targeted advance care planning. J Alzheimer's Dis 2016; 53: 117-25.

16. Chamandy N, Wolfson C. Underlying cause of death in demented and non-demented elderly Canadians. Neuroepidemiology 2005; 25: 75-84.

17. Brodaty H, McGilchrist C, Harris L, Peters KE. Time until institutionalization and death in patients with dementia. Role of caregiver training and risk factors. Arch Neurol 1993; 50: 643-50.

18. Brunnström HR, Englund EM. Cause of death in patients with dementia disorders. Eur J Neurol 2009; 16: 488-92.

19. Phelan EA, Borson S, Grothaus L, Balch S, Larson EB. Association of incident dementia with hospitalizations. JAMA 2012; 307: 165-72.

20. Wardlaw JM, Allerhand M, Doubal FN. Vascular risk factors, large-artery atheroma, and brain white matter hyperintensities. Neurology 2014; 82: 1331-8.

21. Norrving B. Long-term prognosis after lacunar infarction. Lancet Neurol 2003; 2: 238-45.

22. Eriksson SE, Olsson JE. Survival and recurrent strokes in patients with different subtypes of stroke: a 14-year follow-up study. Cerebrovasc Dis 2001; 12: 171-80.

23. Kolominsky-Rabas P, Weber M, Gefeller O. Epidemiology of ischemic stroke subtypes according to TOAST criteria: incidence, recurrence, and long-term survival in ischemic stroke subtypes: a population-based study. Stroke 2001; 32: 2735-40.

24. Jackson C, Sudlow C. Comparing risks of death and recurrent vascular events between lacunar and non-lacunar infarction. Brain 2005; 128: 2507-17.

25. The SPS3 Study Group. Blood-pressure targets in patients with recent lacunar stroke: the SPS3 randomised trial. Lancet 2013; 382: 507-15.

26. Arsava EM, Rahman R, Rosand J. Severity of leukoaraiosis correlates with clinical outcome after ischemic stroke. Neurology 2009; 72: 1403-10.

27. Grefkes C, Nowak DA, Eickhoff SB. Cortical connectivity after subcortical stroke assessed with functional magnetic resonance imaging. Ann Neurol 2008; 63: 236-46.

28. Debette S, Markus HS. The clinical importance of white matter hyperintensities on brain magnetic resonance imaging: systematic review and metanalysis. BMJ 2010; 341: 3666.

29. Kim BJ, Lee SH. Prognostic impact of cerebral small vessel disease on stroke outcome. J Stroke 2015; 17: 101-10.

30. Charidimou A, Kakar P, Fox Z. Cerebral microbleeds and recurrent stroke risk: systematic review and meta-analysis of prospective ischemic stroke and transient ischemic attack cohorts. Stroke 2013; 44: 995-1001.

31. Yamauchi H, Fukuda H, Oyanagi C. Significance of white matter high intensity lesions as a predictor of stroke from arteriolosclerosis. J Neurol Neurosurg Psychiatry 2002; 72: 576-82

32. Inzitari D, Simoni M, Pracucci G; Ladis Study Group. Risk of rapid global functional decline in elderly patients with severe cerebral age-related white matter changes: the Ladis Study. Arch Intern Med 2007; 167: 81-8. 